\title{
МАТЕМАТИЧЕСКОЕ МОДЕЛИРОВАНИЕ ПРОЦЕССОВ ТЕПЛОПЕРЕНОСА ПРИ РАБОТЕ ТЕПЛОНАСОСНЫХ СИСТЕМ ИСПОЛЬЗОВАНИЯ ГЕОТЕРМАЛЬНОЙ ЭНЕРГИИ
}

\author{
Максимов Вячеслав Иванович', \\ elf@tpu.ru \\ Салум Амер', \\ amer@tpu.ru \\ 1 Национальный исследовательский Томский политехнический университет, \\ Россия, 634050, г. Томск, пр. Ленина, 30.
}

\begin{abstract}
Актуальность. Использование теплонасосных установок для отопления вместо традиционных систем, которые получают энергию в процессе сжигания различных видов топлива, имеет ряд экологических и экономических преимуществ. Тепловые насосы могут получать энергию из воздуха, грунта и воды. Их сферы применения разнообразны: горячее водоснабжение и кондиционирование помещений, нагрев и охлаждение воды для различных нужд, сушки/осушения воздуха, производства пара, испарения, дистилляции. При применении природных водоёмов (озёра, пруды, водохранилища) в качестве низкопотенциального источника энергии теплонасосных установок на поверхности трубки испарителя образуется лёд. Важно рассматривать закономерности и характеристики процессов теплообмена между водой и трубкой испарителя при образовании льда на её поверхности.

Цель: математическое моделирование нестационарного конвективного теплообмена между водой и трубками испарителя теплонасосных установок в условиях формирования льда на их поверхности.

Объект: теплообменник испарителя теплового насоса, погружённый в воду

Методы: численное решение задач конвективного теплообмена в условиях фазового перехода воды методом конечных элементов в среде COMSOL.

Результаты. Установлены закономерности нестационарного конвективного теплопереноса вблизи трубок испарителя водяного теплового насоса с температурой, при которой образуется лёд на их поверхности. Показана необходимость учета влияния термогравитационной конвекции в воде на тепловой поток и процесс образования льда на поверхности трубки испарителя теплонасосной установки. Получены зависимости числа Нуссельта от характеристики конвективного теплообмена в воде (чисел Рэлея, Фурье и (тефана). Выявлено, что перепад температур в воде вблизи трубки увеличивался при уменьшении глубины её расположения относительно поверхности водного источника при показателях температур воды выше 277 К. При температурах воды ниже 277 К тепловой поток достигал максимального значения у поверхности трубки, которая находилась глубже.
\end{abstract}

\section{Ключевые слова:}

Термогравитационная конвекция, образование льда, водяной тепловой насос, фазовый переход, низкопотенциальный источник энергии.

\section{Введение}

Использование геотермальных ресурсов в последнее время представляет собой одну из актуальных задач [1-3], но для ее успешного решения необходимо применять новые технологии [4-6]. Особенно перспективным, по мнению многих специалистов [7-9], становится применение в локальных системах теплоснабжения теплонасосных установок (ТНУ) с целью использования геотермальных водных ресурсов - источников теплоты. Применение ТНУ для теплоснабжения как самостоятельной технологии или комбинированной с различными источниками энергии [10] увеличивает экономичность и экологичность системы отопления [11], технология выработки теплоты с использованием установки имеет ряд особенностей, которые осложняют применение ТНУ в холодные времена года [12]. Компрессорные тепловые насосы обладают высоким коэффициентом преобразования энергии, так как при получении тепла из низкопотенциального источника за счёт испарения фреона происходит увеличение его давления и температуры в компрессоре, после чего нагрев теплоносителя в процессе перехода хладагента из газообразного состояния в жидкое в конденсаторе.
Но высокая эффективность этого процесса возможна только в относительно узком интервале температур источника низкопотенциального тепла. При уменьшении этой температуры интенсивность испарения фреона замедляется, в результате чего значительно снижается эффективность работы ТНУ $[13,14]$.

Недостатком применения непроточных водоёмов в качестве источника энергии ТНУ является их низкая температура в холодное время года и, соответственно, обмерзание трубок испарителя, что приводит к существенному снижению характеристик работы теплового насоса в целом. Для повышения энергоэффективности таких систем использования геотермальной энергии, эксплуатируемых при низких температурах, необходима оценка интенсивности процессов обмерзания трубок теплообменника - испарителя ТНУ в условиях пониженной температуры омывающего их низкопотенциального источника тепла - холодной воды. Экспериментальное исследование процессов теплопереноса в слое воды вокруг трубок испарителя ТНУ затруднено по целому ряду причин (замкнутое пространство, малые характерные размеры, образование льда и др.). Основным методом анализа 
характеристик процессов теплопереноса в малой окрестности трубок испарителя (температур, тепловых потоков, толщин слоя льда) является математическое моделирование.

Целью работы является математическое моделирование процессов теплопереноса в воде, окружающей трубки испарителя ТНУ, и образования льда на их поверхности.

\section{Постановка задачи и метод решения}

Экспериментальные исследования [15] показали, что перенос теплоты в пространстве между трубками испарителя работающего теплового насоса и водой осуществляется за счет теплопроводности и термогравитационной конвекции. Поэтому при постановке задачи теплопереноса вокруг трубок испарителя необходимо учитывать эти два механизма переноса теплоты. Рассматривалась двумерная прямоугольная область с водой, в которой располагались три трубки испарителя ТНУ (рис. 1). В общем случае их может быть и больше, но для обоснования выводов об основных закономерностях исследуемых процессов конвекции и кондукции достаточно трех.



Pис. 1. Область решения задачи: 1 - вода; 2 - трубки испарите ля ТНУ; 3- условные гранииы области решения, выделенные в водоеме с непроточной водой

Fig. 1 . Studied area: 1 is the water; 2 are the evaporator heat pump pipes; 3 are the studied area boundary conditions, which represent a reservoir with non-moving water

Решение задачи теплопереноса в области, представляющей собой типичный фрагмент рабочей зоны испарителя теплового насоса, проведено в рамках модели, разработанной авторами [16]. Решалась система нелинейных нестационарных дифференциальных уравнений (1)-(4) (уравнения сохранения массы, импульса и энергии) аналогичная приведенной в $[16,17]$ :

$$
\nabla U=0 \text {, }
$$

$$
\rho\left(\frac{\partial U}{\partial \tau}+U \nabla U\right)-\left(\mu \nabla\left(\nabla U+(\nabla U)^{T}\right)-\rho(T) g=0,(2)\right.
$$

$$
\begin{gathered}
\rho C_{P} \frac{\partial T}{\partial \tau}+\rho C_{P} U \nabla T-\nabla \lambda \nabla T=0, \\
\rho=\rho_{i}(1-\alpha(T))+\rho_{w} \alpha(T),
\end{gathered}
$$

где $T$ - температура, $K$; $U$ - скорость, м/с; $\tau$ - время, с; $\mu$ - динамическая вязкость, $\mathrm{H} \cdot c / \mathrm{m}^{2} ; \rho_{i}, \rho_{w}-$ плотность льда и воды, соответственно, кг $/ \mathrm{m}^{3} ; C_{P, i}$, $C_{P, w}$ - удельная теплоемкость льда и воды, соответственно, Дж/кг $К ; g$ - ускорение свободного падения, м $/ \mathrm{c}^{2} ; \alpha(T)$ - фазовый индикатор (для твердого вещества $\alpha(T)=0$, для жидкости $\alpha(T)=1)$.

С целью учета влияния фазового перехода на теплоёмкость среды использовалось выражение (5), (6) [18]:

$$
\begin{gathered}
C_{P}=C_{P, i}(1-\alpha(T))+C_{P, w} \alpha(T)+L_{1 \rightarrow w} \frac{\partial \alpha_{m}}{\partial T}, \\
\alpha_{m}=\frac{1}{2} \frac{\rho_{i}(1-\alpha(T))-\rho_{w} \alpha(T)}{\rho_{i}(1-\alpha(T))+\rho_{w} \alpha(T)} .
\end{gathered}
$$

Теплопроводность вычислялась с учетом возможного изменения объёмных долей компонентов (7):

$$
\lambda=\lambda_{i}(1-\alpha(T))+\lambda_{w} \alpha(T),
$$

где $\lambda_{i}, \lambda_{w}-$ теплопроводность льда и воды, соответственно, (Вт/м.К); $L_{i \rightarrow w}-$ скрытая теплота плавления льда, кДж/кг; $\frac{\partial \alpha_{m}}{\partial T}-$ импульс Дирака, который является производной от функции Хевисайда. Функция Хевисайда равна нулю в случае отрицательных показателей аргумента и - одному при положительных (эти функции указывают на переход из одного фазового состояния в другое при температуре фазового перехода $273 \mathrm{~K}$ ).

При проведении численного моделирования предполагалось, что теплофизические свойства воды (за исключением плотности) не зависят от температуры, а режим течения принят ламинарным.

Начальные условия для системы уравнений (8)-(10):

$$
u=0 \mathrm{~m} / \mathrm{c}, v=0 \mathrm{M} / \mathrm{c}, T_{w}=280 \mathrm{~K},
$$

$u, v$ - компоненты скорости на оси $O x, O y$, соответственно; $T_{w}$ - средняя температура воды, $\mathrm{K}$.

Граничные условия (9), (10):

На внешних границах области решения:

$$
u=0 \mathrm{~m} / \mathrm{c}, v=0 \mathrm{~m} / \mathrm{c}, \frac{\partial \dot{O}}{\partial x}=\frac{\partial \dot{O}}{\partial y}=0 .
$$

На поверхностях трубок испарителя (10):

$$
u=0 \mathrm{M} / \mathrm{c}, v=0 \mathrm{~m} / \mathrm{c}, T_{p}=263 \mathrm{~K},
$$

$T_{p}$ - температура поверхности трубки испарителя со стороны воды, $\mathrm{K}$.

Численные исследования выполнялись при следующих значениях основных параметров:

- Теплофизические: $\lambda_{w}=0,56 \mathrm{BT} / \mathrm{M} \cdot \mathrm{K} ; \lambda_{i}=2,25 \mathrm{BT} / \mathrm{M} \cdot \mathrm{K} ;$ $C_{P, w}=4,2$ Дж/кг.К; $C_{P, i}=2,22$ Дж/кг $\mathrm{K} ;$ $\mu=0,0015 \mathrm{H} \cdot \mathrm{c} / \mathrm{M}^{2} ; L_{i \rightarrow w}=333,5 \kappa$ КЖ $/$

- Геометрические:

$h 1=h 4=10 \mathrm{Mm} ; h 2=h 3=40 \mathrm{Mm} ; h=100 \mathrm{MM}$; $l=100 \mathrm{Mm} ; l 1=l 2=50 \mathrm{Mm} ; d=9,54 \mathrm{Mm}$. 
Система уравнений с соответствующими краевыми условиями (1-10) решена в среде COMSOL [18] методом конечных элементов. Использовался ряд модулей: однофазного ламинарного потока «Laminar flow.spf» и теплопередачи в жидкости «Heat transfer in liquid.htf». При проведении вычислений внутри каждого модуля работали несколько фундаментальных компонентов: гравитация в модуле "Laminar flow.spf» и изменение фазового состояния в модуле "Heat transfer in liquid.htf». В модуле (Laminar flow.spf) вязкость варьировалась от её значения для воды при температуре $293 \mathrm{~K}$ до «бесконечно большого» в зависимости от температуры. При моделировании по аналогии с [16] рассматривались две среды: жидкость (вода) и твердое тело (лёд). Учитывалось, что вязкость льда существенно больше её значения для жидкости. В начальный момент времени температура во всех точках области решения составляла $T_{w}=280$ К. При этом вода считалась неподвижной. Во время моделирования температура поверхности трубки испарителя имела постоянное значение и составляла 263 К (температура кипения хладагента). На сторонах квадрата приняты условия «неразрывности» $\frac{\partial T}{\partial x}=\frac{\partial T}{\partial y}=0$, соответствующие границам, до которых не доходит «тепловая волна» [18].

Применялась неравномерная (сгущающаяся) разностная сетка. Для повышения точности вычислений использовался неравномерный шаг по времени $\Delta \tau$ (от 0,5 до $0,1 \mathrm{c})$. Характерное время моделирования составляло 5000 с. Выбран параллельный итерационный прямой решатель (PARDISO). В результате численных исследований определены основные характеристики процесса: поля скоростей и температур (рис. 2-5).


Рис. 2. Поля температур в разные моменты времени при размещении трех трубок испарителя в области решения: а) $\tau=100$ с; б) $\tau=300 c$; в) $\tau=500 c$; г) $\tau=1000 c$

Fig. 2. Temperature field in different moments in the case when three pipes are located in the studied area: a) $\tau=100 \mathrm{sec} ; b) \tau=300 \mathrm{sec} ; c$ ) $\tau=500 \mathrm{sec}$; $d=1000 \mathrm{sec}$ 


\section{Результаты численного моделирования}

Представлены распределения температур в зоне испарителя (рис. 2) с тремя трубками, расположенными на одинаковом расстоянии друг от друга (рис. 1)

Анализ результатов вычислений показывает (рис. 2), что в первые 200 с работы ТНУ максимальная температура воды $280 \mathrm{~K}$ сохранялась в верхней части области решения вблизи верхней трубки. Охлажденные слои воды перемещались вниз. При снижении температуры воды до $277 \mathrm{~K}$ (соответствует моменту времени 300 с) в результате работы испарителя направление температурной стратификации изменялось на противоположное: холодная вода поднималась вверх, а слои воды с температурой 277 К перемещались вниз. С ростом



$a / a$



$B / c$ времени вода продолжала охлаждаться за счет теплообмена с холодными фреоновыми трубками. При $\tau=1000$ с температура воды снижалась до 273 К, и конвективное движение прекращалось. В первые 100 с работы ТНУ на нижней части верхней трубки формировался слой льда большей толщины, чем на верхней (рис. 3). Это объясняется тем, что со стороны нижней части верхней трубки температура воды первые 100 с была ниже, чем в области верхней части (рис. 2). На стенке нижней трубки наблюдалась противоположная картина: толщина образовавшегося льда была выше на её верхней части. По истечении 600 с толщина слоя образовавшегося льда продолжала равномерно увеличиваться на теплообменных поверхностях всех трубок (рис. 3).

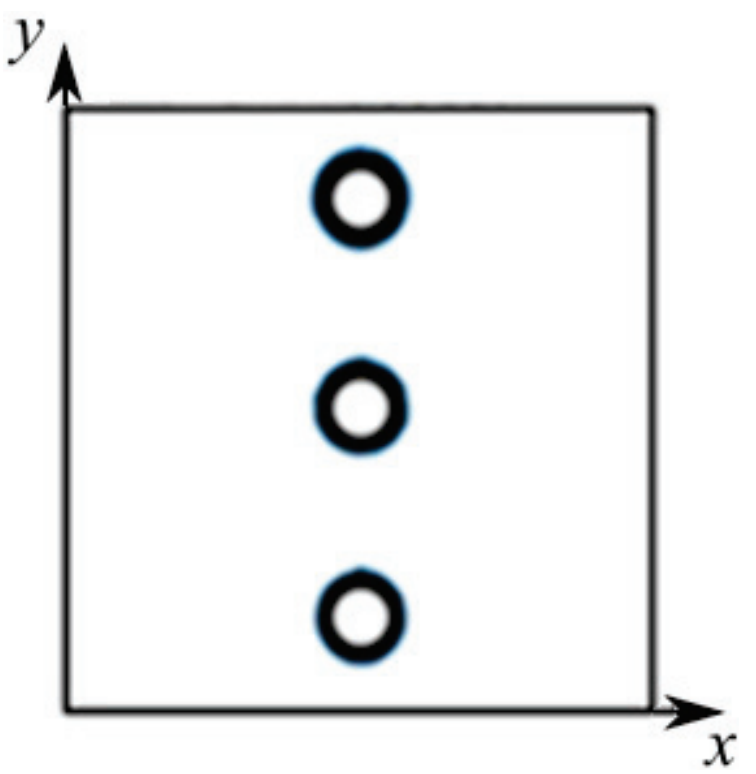

$\sigma / b$

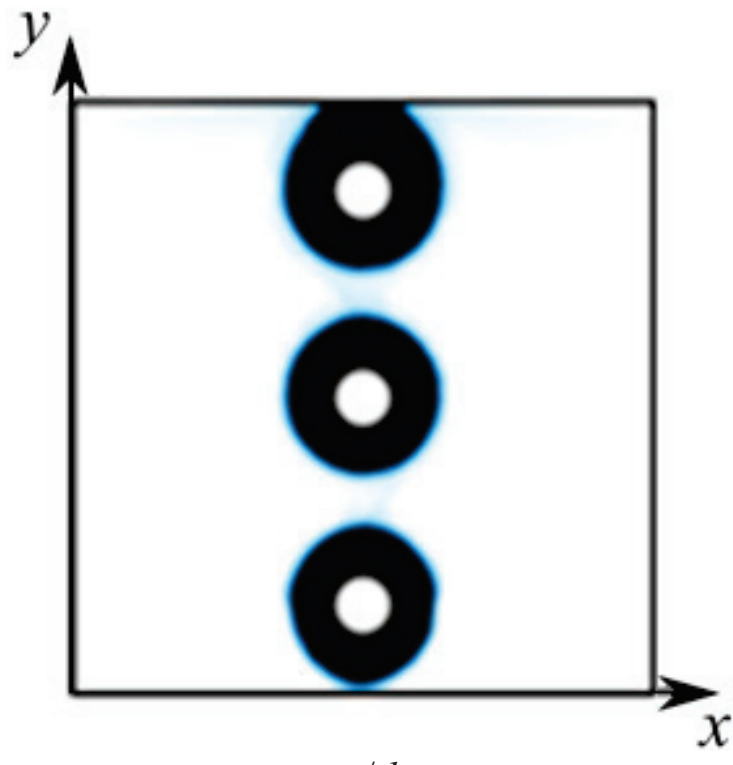

$2 / d$

Pис. 3. Изменение толщины льда на поверхностах трубок испарителя со временем: а) $\tau=100$ c; б) $\tau=700$ c; в) $\tau=1500$ c; г) $\tau=5000$ c

Fig. 3. Ice thickness changes on the evaporator pipes surfaces with time: a) $\tau=100 \mathrm{sec}$; $b) \tau=700 \mathrm{sec}$; c) $\tau=1500 \mathrm{sec}$; d) $\tau=5000 \mathrm{sec}$ 


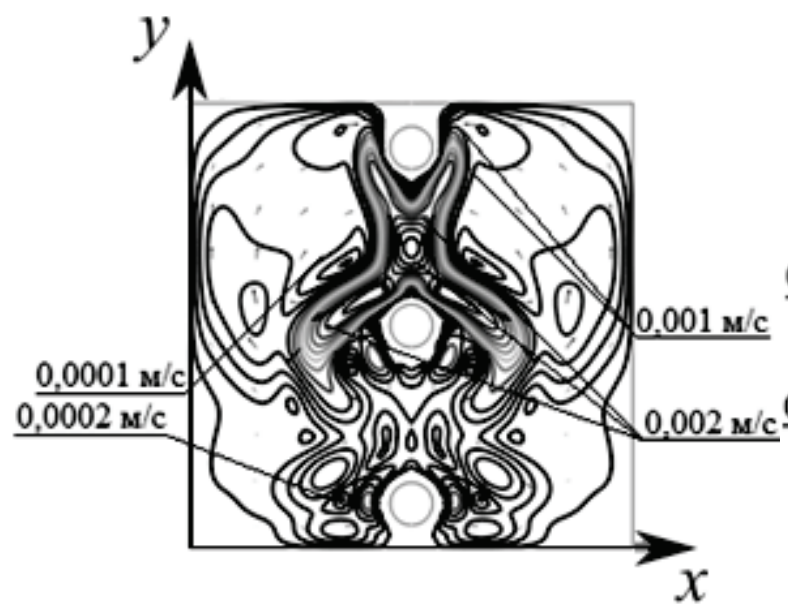

$a / a$

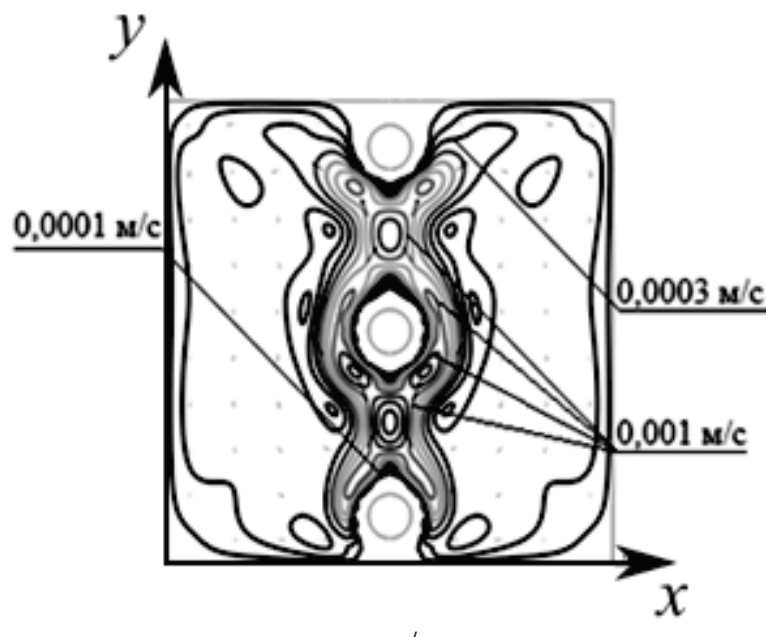

$B / c$

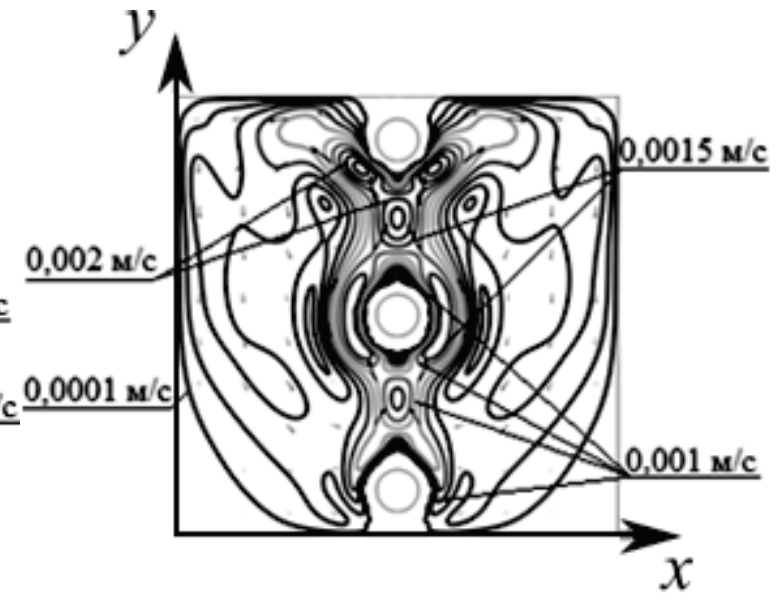

$\sigma / b$

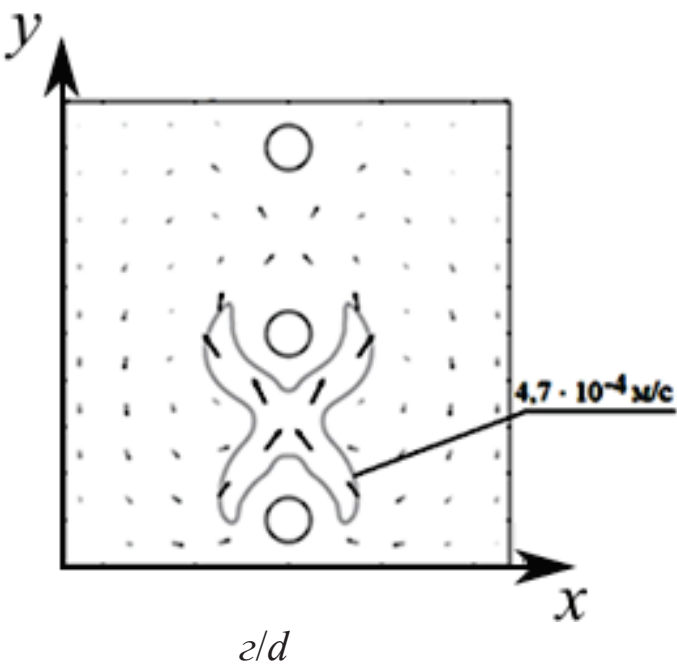

Pис. 4. Линии постоянных скоростей течения воды вблизи поверхности трубок испарителя в разные моменты времени: а) $\tau=100$ с; б) $\tau=300 c$; в) $\tau=500 c$; г) $\tau=1000 c$

Fig. 4. Water velocity lines around the evaporator pipes in different moments of time: a) $\tau=100 \mathrm{sec}$; b) $\tau=300 \mathrm{sec} ; \mathrm{c}$ ) $\tau=500 \mathrm{sec}$; d) $\tau=1000 \mathrm{sec}$

Заметны изменения численных значений и направлений скорости движения охлаждаемой воды при изменении её температуры и, соответственно, плотности (рис. 4). Наиболее интенсивное движение под влиянием подъёмной силы имеет место в области расположения трубок испарителя. При увеличении расстояния от их поверхности по координатным направлениям $x$ и $y$ конвективное перемещение воды становилось более медленным. Средняя скорость термогравитационного течения воды в области трубок испарителя составляла 0,001 м/с при температурах 277-280 K.

Градиенты температур приводят к образованию двух крупных вихрей в рассматриваемой области решения (рис. 5). По истечении 1000 с температура воды выравнивается по всему объему и становится равной $273 \mathrm{~K}$, конвективное перемещение воды, по существу, прекращается.

Показано изменение средней толщины слоя льда, образующегося на поверхностях трубок испарителя, со временем (рис. 6). Заметно, что в первые
300 с работы ТНУ скорость образования льда на поверхностях всех трех трубок практически одинакова. С ростом времени толщина слоя льда увеличивается быстрее на трубках, расположенных ближе к верхней границе области, т. к. после 300 с работы в верхней части области решения сосредотачиваются слои воды с более низкой температурой (рис. 2). Это способствует более быстрому обледенению верхней трубки.

Показаны изменения теплового потока между трубками с хладоном и водой вблизи поверхностей трубок (рис. 7). В интервале времени до 300 с тепловой поток от воды к верхней трубке незначительно больше, чем к нижней трубке.

Это объясняется тем, что в первые 300 с работы ТНУ тёплые слои воды располагаются ближе к верхней трубке. В интервале времени $300 \leq \tau \leq 1000$ с изменяется направление температурной стратификации, и слои с более низкой температурой поднимаются вверх, тепловой поток между водой и нижней трубкой становится боль- 


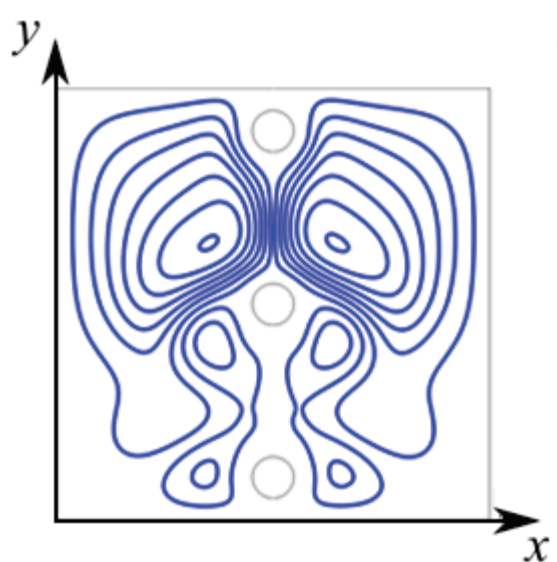

$a / a$

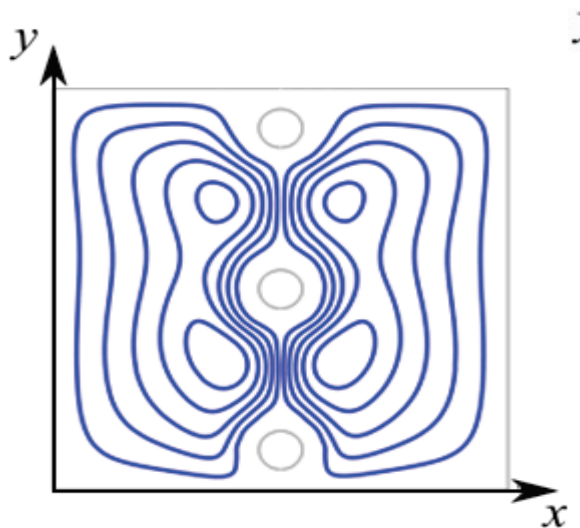

$B / c$

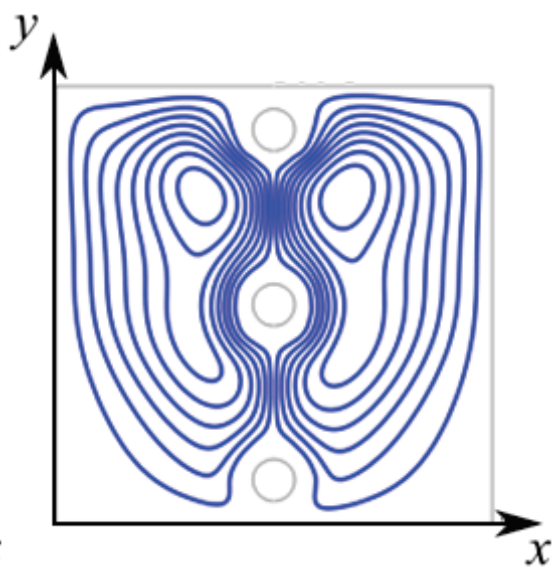

$\sigma / b$

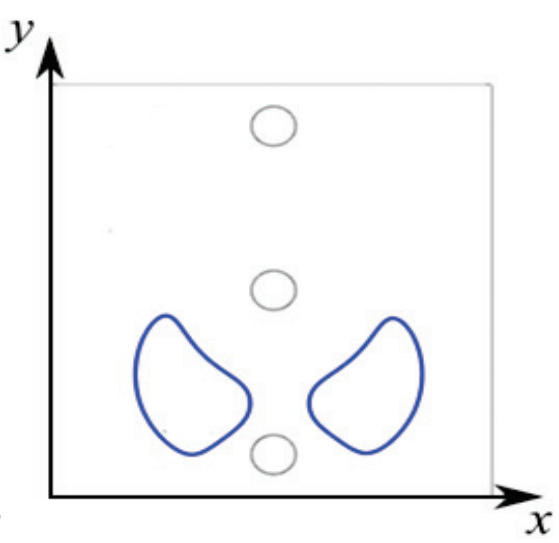

$2 / d$

Pис. 5. Линии тока воды в разные моменты времени: а) $\tau=100$; б) $\tau=300$ c; в) $\tau=500$ c; г) $\tau=1000$ c

Fig. 5. Water current lines in different moments from modeling time: a) $\tau=100 \mathrm{sec}$; b) $\tau=300 \mathrm{sec}$; $) \tau=500 \mathrm{sec}$; d) $\tau=1000 \mathrm{sec}$

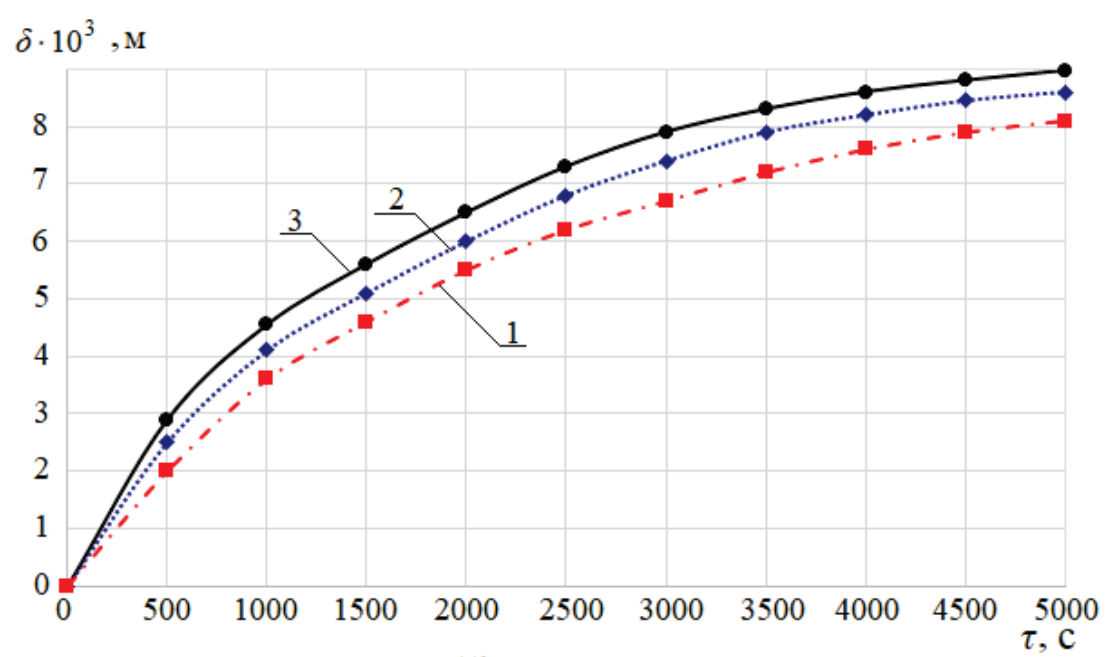

Pис. 6. Изменения толщины льда на поверхности трубок испарителя со временем: 1 - нижняя; 2 - центральная; 3 - верхняя

Fig. 6. Dependence of ice thickness formed on the evaporator pipe surface with time: 1 - for lower pipe; 2 - for central pipe; 3 - for higher pipe

ше. Спустя 1000 с температура воды во всем объеме снижается до $273 \mathrm{~K}$, что соответствует условиям кристаллизации воды на стенке трубки испарителя (рис. 7).

\section{Заключение}

Решение задачи нестационарного теплопереноса в воде, окружающей трубки испарителя ТНУ, и образования льда на их поверхности даёт возмож- 




Рис. 7. Изленения во времени теплового потока между водой и трубками испарителя: 1 - нижняя; 2 - иентральная; 3 - верхняя

Fig. 7. Heat flux between the water and the evaporator pipe: 1 - for lower pipe; 2 - for central pipe; 3 - for higher pipe

ность оценить интенсивность процессов обмерзания трубок теплообменника - испарителя ТНУ - в условиях пониженной температуры омывающего их низкопотенциального источника тепла - холодной воды.

Полученные в результате математического моделирования данные позволяют оценить энергоэффективность систем ТНУ, эксплуатируемых при низких температурах воды, и дать рекомендации

\section{СПИСОК ЛИТЕРАТУРЫ}

1. Todoran T.P., Balan M. C. Long term behavior of a geothermal heat pump with oversized horizontal collector // Energy and Buildings. - 2016. - V. 133. - P. 799-809. URL: https://www.sciencedirect.com/science/article/pii/S0378778816312889 (дата обращения: 15.02.2019).

2. Evaporation heat transfer and pressure drop for geothermal heat pumps working with refrigerants R134a and R407C / Kh.R. Dione, H. Louahlia, M. Mariona, J.L. Berçaits // International Communications in Heat and Mass Transfer. - 2018. V. 93. - P. 1-10. URL: https://www.sciencedirect.com/science/ article/pii/S0735193318300162 (дата обращения: 15.02.2019).

3. Харченко В.В., Сычев А.О. Оптимизация низкотемпературного контура теплонасосной установки на основе теплоты поверхностных вод // Альтернативная энергетика и экология. 2013. - № 7 (129). - C. 31-36. URL: https://elibrary.ru/item.asp? id=19403468 (дата обращения: 15.02.2019).

4. Гершкович В.Ф. От централизованного теплоснабжения - к тепловым насосам // Энергосбережение. - 2010. - № 3. C. 24-28. URL: https://www.abok.ru/for_spec/articles.php? nid=4558 (дата обращения: 15.02 .2019 ).

5. Zhou C., Ni L., Yao Y. Heat transfer analysis of multi-row helically coiled tube heat exchangers for surface water-source heat pump // Energy. - 2018. - V. 163. - P. 1032-1049. URL: https://www.sciencedirect.com/science/article/abs/pii/ S0360544218317262 (дата обращения: 15.02.2019).

6. Analysis on Several Heat Pump Applications in Large Public Buildings / L. Shengchun, Z. Wenkai, D. Ziteng, S. Gang // Journal of Building Construction and Planning Research Technology. 2015. - № 3. - P.136-148. URL: https://file.scirp.org/pdf/ JBCPR 2015092215345661.pdf (дата обращения: 15.02.2019).

7. Сычев А.О., Харченко В.В. Пути повышения технико-экономических показателей теплонасосных установок, использующих теплоту поверхностных вод // Альтернативная энергети- по повышению эффективности их работы в условиях осеннего, зимнего и весеннего периодов года.

Исследование проведено в рамках програмлы повышения конкурентоспособности Национального исследовательского Томского политехнического университета среди ведущих мировых научно-образовательных иентров (Госзадание "Наука» 8.13264.2018/8.9, проект ВИУИШЭ-300/2018).

ка и экология. - 2015. - № 10-11. - C. 84-90. URL: https:// www.isjaee.com/jour/article/view/123?locale=ru_RU (дата обращения: 15.02.2019).

8. Maksimov V.I., Saloum A. Convection influence on ice formation process on the evaporator heat pump pipes // Smart Grids 2017: The Fifth International Youth Forum: MATEC Web of Conferences. Tomsk, Russia, 22-23 April, 2017. - V. 141. - 5 p. URL: https:// www.matec-conferences.org/articles/matecconf/pdf/ 2017/55/matecconf_smartgrids2017_01001.pdf (дата обращения: 15.02.2019).

9. Maksimov V.I., Nagornova T.A., Chernyshev V.S. Conditions and characteristics of water crystallization on the working surface of evaporator heat pumps in reservoirs with low temperatures // Heat and Mass Transfer in the Thermal Control System of Technical and Technological Energy: MATEC Web of Conferences. Tomsk, Russia, 22-23 April, 2015. - V. 23. - 8 p. URL: https:// www.matec-conferences.org/articles/matecconf/pdf/2015/04/ matecconf tsotr2015 01051.pdf (дата обращения: 15.02.2019).

10. A study on the evaluation of the annual energy consumption for a geothermal heat pump system with open loop and closed loop ground heat exchangers / S. Baohen, K.H. Lee, S. Cho, J.M. Choi // International Journal of Air-Conditioning and Refrigeration. - 2017. V. 25. - № (3). - 35 p. URL: https://www.worldscientific.com/doi/ 10.1142/S2010132517500249 (дата обращения: 15.02.2019).

11. Liu Z., Tan H., Li Z. Heating and Cooling Performances of RiverWater Source Heat Pump System for Energy Station in Shanghai // Procedia Energy. - 2017. - V. 205. - P. 4074-4081. URL: https://www.sciencedirect.com/sdfe/pdf/download/read/noindex/pii/S1877705817344569/1-s2.0-S1877705817344569main.pdf (дата обращения: 15.02.2019).

12. Amoabeng K.O., Lee K.H. A study on the performance characteristics of a testing facility for a water - to - water heat pump // International Journal of Refrigeration. - 2018. - V. 86. P. 113-126. URL: https://www.sciencedirect.com/science/article/pii/S0140700717304565 (дата обращения: 15.02.2019). 
13. Zou S., Xie X. Simplified model for coefficient of performance calculation of surface water source heat pump // Applied thermal energy. - 2017. - V. 112. - P. 201-207. URL: https://www.sciencedirect.com/science/article/pii/S135943111632378X (дата обращения: 15.02.2019).

14. Schibuola L., Scarpa M. Experimental analysis of the performances of a surface water source heat pump // Energy and Buildings. - 2015. - V. 113. - P. 182-188. URL: https://www.sciencedirect.com/science/article/pii/S0378778815304849 (дата обращения: 15.02.2019).

15. Hansen G.M. Experimental Testing and Analysis of Spiral-Helical Surface Water Heat Exchanger Configurations: a thesis of the degree Bachelor of Science in Mechanical Engineering. - Oklahoma, 2011. - 171 p. URL: https://hvac.okstate.edu/sites/default/files/pubs/theses/MS/Garrett $\%$ 20Michael\% $20 \mathrm{Han}$ sen $\%$ 20Masters\% 20Thesis\% 202011.pdf (дата обращения: 15.02.2019)

16. Bourisli R., Alshayji A. Natural convection effects on the solidification in cylinders at different filling percentages // The
2017 COMSOL Conference in Rotterdam. - Rotterdam, Niederlande, 18-20 September, 2017. - 5 p. URL: https://www.comsol.com/paper/download/442131/bourisli_abstract.pdf (дата обращения: 15.02.2019).

17. Analysis of the icing and melting process in a coil heat exchanger/ Y. Li, C.Yang, Z.Yan, B.Guo, H.Yuan, J. Zhao, N. Mei // Energy Procedia. - 2017. - V. 136. - P. 450-455. URL: https://www.sciencedirect.com/sdfe/pdf/download/read/noin$\mathrm{dex} / \mathrm{pii} / \mathrm{S} 1876610217352529 / 1$-s2.0-S1876610217352529main.pdf (дата обращения: 15.02.2019).

18. Morton A.C. Assessing the performance of a reservoir-based water source heat pump: a thesis of the degree Master of Science. Glasgow, Scotland, 2013. - 89 p. URL: http://www.esru.strath.ac.uk/Documents/MSc_2013/Morton.pdf (дата обращения: 15.02.2019).

Поступила 26.02.2019 г.

\section{Информация об авторах}

Максимов В.И., кандидат технических наук, доцент научно-образовательного центра им. И.Н. Бутакова Инженерной школы энергетики Национального исследовательского Томского политехнического университета.

Салум А., аспирант научно-образовательного центра им. И.Н. Бутакова Инженерной школы энергетики Национального исследовательского Томского политехнического университета. 
UDC 621.577.001.5

\title{
MATHEMATICAL MODELING OF HEAT TRANSFER BY OPERATION OF GEOTHERMAL HEAT PUMPS
}

\author{
Vyacheslav I. Maksimov', \\ elf@tpu.ru
}

\author{
Amer Saloum ${ }^{1}$, \\ amer@tpu.ru
National Research Tomsk Polytechnic University, 30, Lenin Avenue, Tomsk, 634050, Russia.

The relevance. The use of heat pumps to provide heat instead of traditional systems, which get energy from the burning of different fossil fuel kinds, has a number of environmental and economic benefits. Heat pumps can use air, ground, and water as an energy source. They can be used for various applications: hot water supply, air conditioning, heating and cooling water for different uses, air drying/dehumidification, vapor production, evaporation, and distillation. By the use of natural water surface (lakes, ponds, reservoirs) as a low-potential heat source for heat pump, ice can be formed on the evaporator pipe surface. It is important to study the heat exchange characteristics between the water and the evaporator pipe undergoing ice formation on its surface.

The main aim of the research is mathematical modeling for non-stationary convective heat exchange between the water and the heat pump evaporator pipes under the conditions of ice formation on their surface.

The object of the research is the heat pump evaporator heat exchanger which is surrounded by water.

The methods of the research are numerical solutions for convective heat transfer problem under the conditions of the water phase change by the use of the finite element method in COMSOL environment.

Results. The authors have established the unsteady convective heat transfer laws near the water source heat pump evaporator pipes with the temperature under water freezing point. In calculations of the heat flux and ice thickness growth rate on the surface of heat pump evaporator pipe, the natural convection in water effect must not be ignored. The authors obtained the dependence of Nusselt number on the natural convection heat exchange characteristics undergoing a phase change (Rayleigh, Fourier and Stefan numbers). It is revealed that the drop rate in water temperature around the pipe increases with the decrease of its depth from the surface of the water source for water temperatures values higher than $277 \mathrm{~K}$. For water temperatures lower than $277 \mathrm{~K}$, the heat flux is maximum around the pipe, which is located deeper.

Key words:

Natural convection, ice formation, water source heat pump, phase change, low-potential heat source.

The research was carried out within the Program of National Research Tomsk Polytechnic University Competitiveness Enhancement among the leading world Research and Educational centers (State Science $« 8.13264 .2018$ / 8.9, project VIUISHE-300/2018).

\section{REFERENCES}

1. Todoran T.P., Balan M. C. Long term behavior of a geothermal heat pump with oversized horizontal collector. Energy and Buildings, 2016, vol. 133, pp. 799-809. Available at: https://www. sciencedirect.com/science/article/pii/S0378778816312889 (accessed 15 February 2019).

2. Kh.R. Dione, H. Louahlia, M. Mariona, J.L. Berçaits. Evaporation heat transfer and pressure drop for geothermal heat pumps working with refrigerants R134a and R407C. International Com munications in Heat and Mass Transfer, 2018, vol. 93, pp. 1-10. Available at: https://www.sciencedirect.com/science/article/pii/S0735193318300162 (accessed 15 February 2019).

3. Kharchenko V.V., Sychev A.0. Optimization of low temperature circuit of surface water heat pump based system. Alternative Energy and Ecology, 2013, no. 7 (129), pp. 31-36. In Rus. Available at: https://elibrary.ru/item.asp?id=19403468 (accessed 15 February 2019).

4. Kershkovich V.F. Ot tsentralizovannogo teplosnabzheniya - $\mathrm{k}$ teplovym nasosam [From district heating to heat pumps]. Energosberezhenie, 2010, no. 3, pp. 24-28. Available at: https:// www.abok.ru/for_spec/articles.php? nid=4558 (accessed 15 February 2019).

5. Zhou C., Ni L., Yao Y. Heat transfer analysis of multi-row helically coiled tube heat exchangers for surface water-source heat pump. Energy, 2018, vol. 163, pp. 1032-1049. Available at: https://www.sciencedirect.com/science/article/abs/pii/ S0360544218317262 (accessed 15 February 2019).
6. Shengchun L., Wenkai Z., Ziteng D., Gang S. Analysis on Several Heat Pump Applications in Large Public Buildings. Journal of Building Construction and Planning Research Technology, 2015, no. 3, pp. 136 -148. Available at: https://file.scirp.org/ pdf/ JBCPR_2015092215345661.pdf (accessed 15 February 2019).

7. Sychev A.0., Kharchenko V.V. Ways to improve technical and economic indicators of a surface water heat pump based systems. Alternative Energy and Ecology, 2015, no. 10-11, pp. 84-90. In Rus. Available at: https://www.isjaee.com/jour/article/view/123?locale=ru_RU (accessed 15 February 2019).

8. Maksimov V.I., Saloum A. Convection influence on ice formation process on the evaporator heat pump pipes. The Fifth International Youth Forum. Smart Grids 2017: MATEC Web of Conferences. Tomsk, Russia, 22-23 April, 2017. Vol. 141, 5 p. available at: https://www.matec-conferences.org/articles/matecconf/ pdf/2017/55/matecconf_smartgrids2017_01001.pdf (accessed 15 February 2019).

9. Maksimov V.I., Nagornova T.A., Chernyshev V.S. Conditions and characteristics of water crystallization on the working surface of evaporator heat pumps in reservoirs with low temperatures. Heat and Mass Transfer in the Thermal Control System of Technical and Technological Energy: MATEC Web of Conferences. Tomsk, Russia, 22-23 April, 2015. Vol. 23, 8 p. Available at: https:// www.matec-conferences.org/articles/matecconf/pdf/2015/04/ matecconf_tsotr2015_01051.pdf (accessed 15 February 2019).

10. Baohen S., Lee K.H., Cho S., Choi J.M. A study on the evaluation of the annual energy consumption for a geothermal heat pump sy- 
stem with open loop and closed loop ground heat exchangers. International Journal of Air-Conditioning and Refrigeration, 2017, vol. 25, no. (3), 35 p. Available at: https://www.worldscientific.com/doi/10.1142/S2010132517500249 (accessed 15 February 2019).

11. Liu Z., Tan H., Li Z. Heating and Cooling Performances of RiverWater Source Heat Pump System for Energy Station in Shanghai. Procedia Energy, 2017, vol. 205, pp. 4074-4081. Available at: https://www.sciencedirect.com/sdfe/pdf/download/read/noindex/pii/S1877705817344569/1-s2.0-S1877705817344569main.pdf (accessed 15 February 2019).

12. Amoabeng K.O., Lee K.H. A study on the performance characteristics of a testing facility for a water - to - water heat pump. International Journal of Refrigeration, 2018, vol. 86, pp. 113-126. Available at: https://www.sciencedirect.com/science/article/pii/S0140700717304565 (accessed 15 February 2019).

13. Zou S., Xie X. Simplified model for coefficient of performance calculation of surface water source heat pump. Applied thermal energy, 2017, vol. 112, pp. 201-207. Available at: https://www. sciencedirect.com/science/article/pii/S135943111632378X (accessed 15 February 2019).

14. Schibuola L., Scarpa M. Experimental analysis of the performances of a surface water source heat pump. Energy and Buildings, 2015, vol. 113, pp. 182-188. Available at: https://www.sciencedirect.com/science/article/pii/S0378778815304849 (accessed 15 February 2019).
15. Hansen G. M. Experimental Testing and Analysis of Spiral-Helical Surface Water Heat Exchanger Configurations. A thesis of the degree Bachelor of Science in Mechanical Engineering. Oklahoma, 2011. 171 p. Available at: https://hvac.okstate.edu/sites/ default/files/pubs/theses/MS/Garrett $\% 20$ Michael $\% 20$ Hansen $\%$ 20Masters $\%$ 20Thesis\% 202011.pdf (accessed 15 February 2019).

16. Bourisli R., Alshayji A. Natural convection effects on the solidification in cylinders at different filling percentages. The 2017 COMSOL Conference in Rotterdam. Rotterdam, Niederlande, 18-20 September, 2017. 5 p. Available at: https://www.comsol.com/paper/download/442131/bourisli_abstract.pdf (accessed 15 February 2019).

17. Li Y., Yang C., Yan Z., Guo B., Yuan H., Zhao J., Mei N. Analysis of the icing and melting process in a coil heat exchanger. Energy Procedia, 2017, vol. 136, pp. 450-455. Available at: https:// www.sciencedirect.com/sdfe/pdf/download/read/noindex/pii/ S1876610217352529/1-s2.0-S1876610217352529-main.pdf (accessed 15 February 2019).

18. Morton A.C. Assessing the performance of a reservoir-based water source heat pump. a thesis of the degree Master of Science. Glasgow, Scotland, 2013. 89 p. Available at: http://www.esru.strath.ac.uk/Documents/MSc_2013/Morton.pdf (accessed 15 February 2019).

Received: 26 February 2019.

\section{Information about the authors}

Vyacheslav I. Maksimov, Cand. Sc., associate professor, National Research Tomsk Polytechnic University.

Amer Saloum, postgraduate student, National Research Tomsk Polytechnic University. 